\title{
Determination of Flow Conditions in Coronary Bifurcation Lesions in the Context of the Medina Classification
}

\author{
Marjan Molavi Zarandi, ${ }^{1}$ Rosaire Mongrain,, ${ }^{1,2}$ and Olivier F. Bertrand ${ }^{3,4}$ \\ ${ }^{1}$ Department of Mechanical Engineering, McGill University, Montreal, QC, Canada H3A OC3 \\ ${ }^{2}$ Department of Cardiovascular Surgery, Montreal Heart Institute, Montreal, QC, Canada H1T 1 C8 \\ ${ }^{3}$ Quebec Heart-Lung Institute, Laval Hospital, Quebec City, QC, Canada G1V 4G5 \\ ${ }^{4}$ Faculty of Medicine, Laval University, Quebec City, QC, Canada G1V OA6
}

Correspondence should be addressed to Rosaire Mongrain, rosaire.mongrain@mcgill.ca

Received 10 January 2012; Accepted 3 March 2012

Academic Editor: Guan Heng Yeoh

Copyright (c) 2012 Marjan Molavi Zarandi et al. This is an open access article distributed under the Creative Commons Attribution License, which permits unrestricted use, distribution, and reproduction in any medium, provided the original work is properly cited.

\begin{abstract}
Coronary artery bifurcation lesions are complex and several classifications are presented to describe them. Recently, the Medina classification has been proposed. This classification uses binary values for characterization of stenosis. Flow conditions according to Medina classification have not been described. In this paper, bifurcation lesions corresponding to anatomical Medina lesion classification are compared on the basis of flow and Wall Shear Stress (WSS). Computational models of healthy and stenosed coronary artery bifurcations $((1,1,1),(0,1,1)$ and $(1,0,1))$ with moderate and severe stenoses of $50 \%$ and $75 \%$ diameter were analyzed. The results showed that, flow conditions vary in bifurcation lesion types according to the clinically-oriented Medina classification. The flow in SB of bifurcation was dependent of the Medina lesion type and was more affected in lesion type ( 1,0 , 1). The magnitudes of WSS on the inner and outer walls of SB of bifurcation lesion $(1,0,1)$ in post-stenotic region and along the arterial wall were smaller than bifurcations lesions $(0,1,1)$ and $(1,1,1)$ respectively. Our results suggest that SB of bifurcation lesion $(1,0,1)$ is more prone to atherosclerosis progression compared to types $(0,1,1)$ and $(1,1,1)$.
\end{abstract}

\section{Introduction}

From clinical practice, it is known that coronary artery bifurcations are regions where the flow is strongly perturbed, and is prone to the development of atherosclerotic lesions. As a definition, bifurcation lesion is a coronary artery narrowing that may involve the proximal main vessel, the distal main vessel, and the side branch [1]. Bifurcation lesions have always represented a major challenge for percutaneous treatment [2-5]. Part of this challenge is related to the variety of coronary lesions located at a bifurcation which present wide range of anatomical morphologies. Currently, there are seven coronary bifurcation lesion classification schemes in the literature [6-13]. These classifications are based on the presence or absence of significant angiographic stenosis within the three vessels of the bifurcation. All the published classifications are very similar in describing a given bifurcation lesion. Different lesion types are named using numbers or letters. Most of the classifications are difficult to remember [6-11]. The first attempt to overcome some of the limitations of previous classifications and simplify these classifications was successfully made by Medina et al. [12]. The classification by Medina is simple and does not need to be memorized even though it provides all the information contained in the other classifications. In this classification, the bifurcation is divided into three segments: the main branch proximal (MBP), the main branch distal (MBD), and the side branch (SB). Any narrowing with critical stenosis of $50 \%$ and above in any segment receives the binary value 1 ; otherwise, a binary value 0 is assigned starting from left to right. The three suffixes are separated by commas. In that context, the Medina classification is essentially an anatomical classification.

Pathologic examination of coronary arteries reveals that atherosclerosis lesions are likely to prevail at places where the vessel is curved, bifurcates, has a junction, a side branch, or 

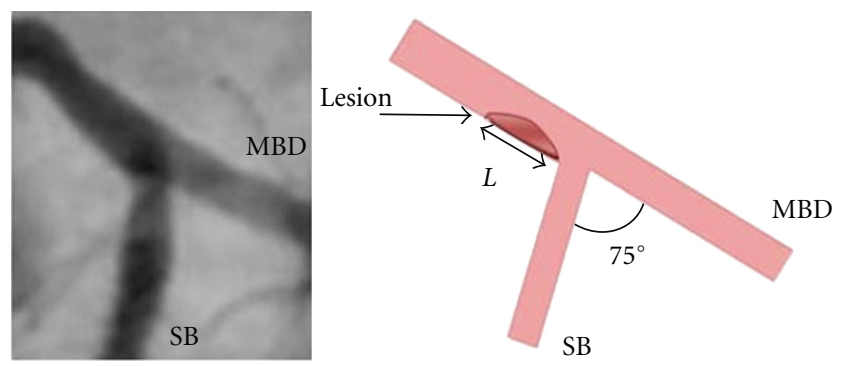

(a)
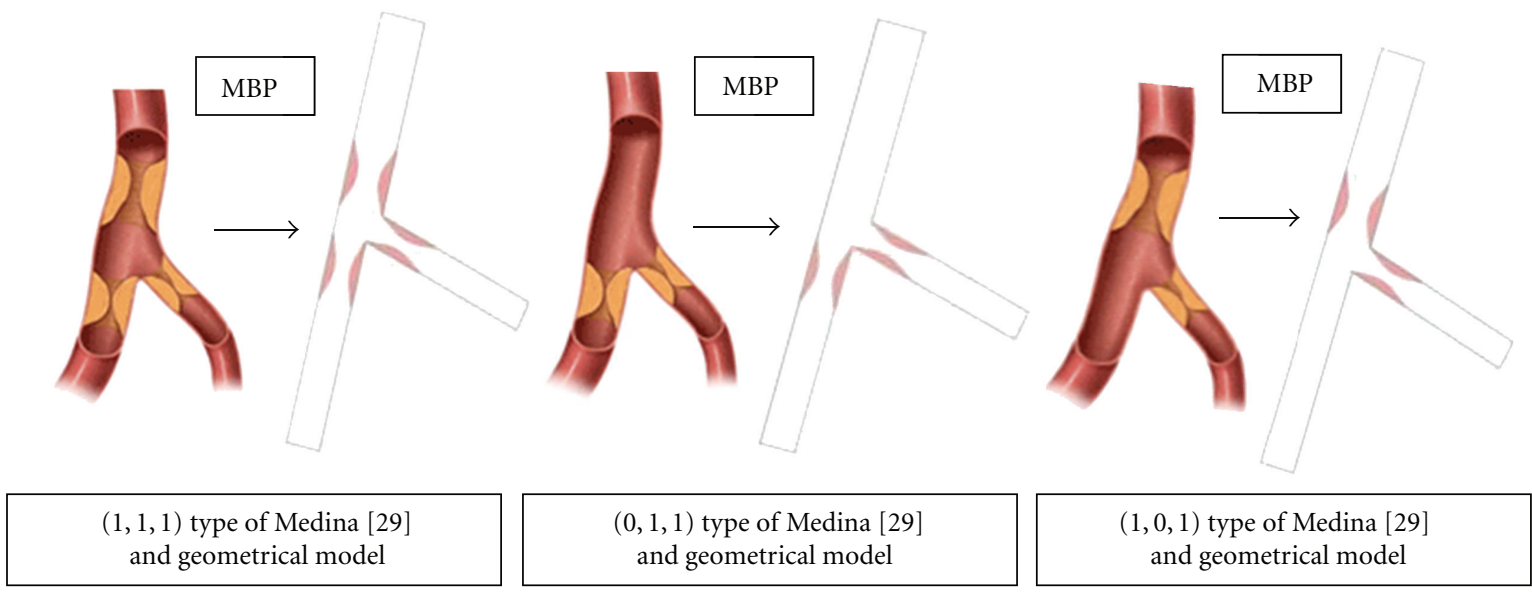

(b)

FIGURE 1: Angiogram of coronary artery bifurcation [26, 29] and corresponding geometrical model (a) and associated Medina lesion classification of coronary artery bifurcation (b).

other sudden change in flow geometry, and when the flow is unsteady [13-16]. Hemodynamic factors play a major role in the initiation and progression of atherosclerosis [17-19]. Studies show that arterial regions exposed to low and nonuniform shear stress are more prone to atherosclerotic lesions development [20-23].

To date, there has been no report studying flow conditions in bifurcation lesions associated with the Medina lesion classification. Using computational fluid dynamics (CFD) analysis, we studied (1) flow conditions in MBP, MBD, and SB, and (2) Wall Shear Stress (WSS) distributions in bifurcations lesions $(1,1,1),(0,1,1)$, and $(1,0,1)$.

\section{Material and Methods}

Hemodynamic analyses were carried out to study the flow conditions and quantify the WSS in coronary artery bifurcation which is of the most important sites of atherosclerotic plaque accumulation [24-26]. The simulation was conducted using COMSOL 3.5 which is dedicated for multiphysics and engineering applications. This software uses the finite-element method to solve the equations that govern blood flow in the computational domain. The computational domain was meshed and the combination of both momentum and continuity equations for transient, Newtonian model of the blood flow is analyzed.

A geometrical model of coronary artery bifurcation was considered to simulate the bifurcation between left main coronary artery (LMCA) and left anterior descending artery (LAD) [26]. Figure 1 shows the geometrical model of twodimensional angiographic projections of the bifurcation (main branch proximal (MBP), main branch distal (MBD), and side branch $(\mathrm{SB})$ ) as well as the bifurcation lesions associated with the Medina lesion classification [3]. In our model, the dimensions of MBP, MBD, and SB are $4 \mathrm{~mm}$, $3.4 \mathrm{~mm}$, and SB $2.7 \mathrm{~mm}$ which are selected based on coronary arteriography data [27]. To have a fully developed flow at the inlet and downstream the stenoses, we considered a branch length $15 \mathrm{D}_{\mathrm{MBP}}$ as a total branch length. The branch lengths for $\mathrm{MBD}$ and $\mathrm{SB}$ are calculated from the centre of the bifurcation to their end, equal to $12 \mathrm{D}_{\mathrm{MBD}}$ and $11_{\mathrm{DSB}}$, respectively. A lesion length (L) of $6 \mathrm{~mm}$ is located at the carina in the considered bifurcation lesions. The angle between the centerline LMCA and LAD as well as the angle between the centerline $\mathrm{MBD}$ and $\mathrm{SB}\left(75^{\circ}\right)$ corresponds to physiological anatomical range [28].

A similar two-dimensional model was recently presented for one stenotic bifurcation case [30].

In the stenosed bifurcation, the word "true" bifurcation lesion is used when stenosis in the proximal and/or distal segment of the main vessel and the side branch is involved [31]. Therefore, according to Medina lesion classification, the bifurcation lesion types $(1,1,1),(0,1,1)$, and $(1,0,1)$ are true bifurcation lesions. The Medina classification seems intuitive in the sense that the most obstructed disease bifurcation is labeled as $(1,1,1)$. A secondary goal of this 


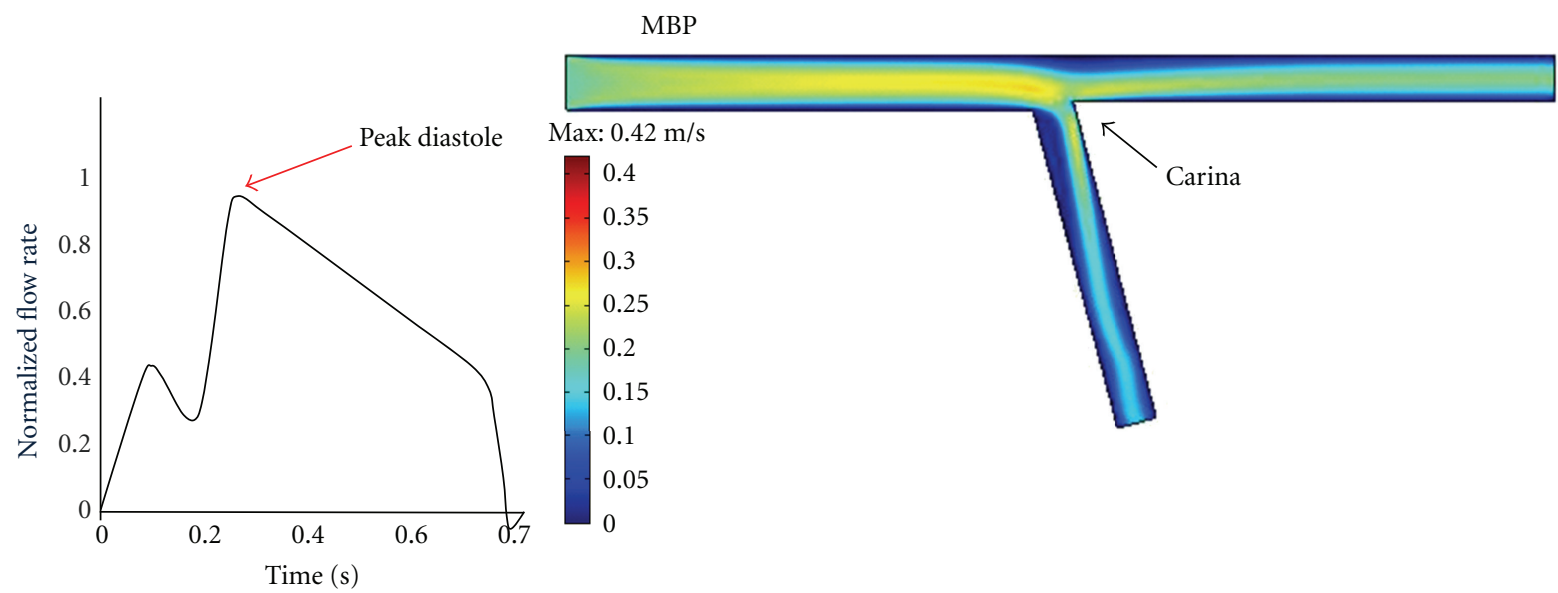

FIgURE 2: Velocity field in coronary artery bifurcation at peak diastole.

study is to assess, on the WSS basis, if the bifurcation type (1, $1,1)$ do correspond to the most severe condition.

For the study of blood flow, we assumed that blood can be represented by an incompressible fluid which is governed by the conservation of mass and the conservation of momentum leading to the Navier-Stokes and the continuity equations:

$$
\begin{gathered}
\rho\left(\frac{\partial \vec{u}}{\partial t}+\vec{u} \cdot \nabla \vec{u}\right)=-\nabla p+\mu\left(\nabla^{2} \vec{u}\right), \\
\nabla \cdot \vec{u}=0,
\end{gathered}
$$

where, $\rho$ denotes the density of the fluid $\left(\mathrm{kg} \mathrm{m}^{-3}\right), \vec{u}$ the velocity vector $\left(\mathrm{ms}^{-1}\right), p$ the pressure $(\mathrm{Pa})$, and $\mu$ the dynamic viscosity of fluid (Pa.s).

Wall shear stress was determined as the product of viscosity $(\mu)$ and the shear rate $(\dot{\gamma})$. The shear rate, in two dimensions is defined according to:

$$
\dot{\gamma}=\sqrt{\left(2\left(\frac{\partial \vec{u}}{\partial x}\right)^{2}+\left(\frac{\partial \vec{u}}{\partial y}+\frac{\partial \vec{v}}{\partial x}\right)^{2}+2\left(\frac{\partial \vec{v}}{\partial y}\right)^{2}\right)}
$$

where $\vec{u}$ and $\vec{v}$ are the velocity components [32].

To solve the governing equations, a set of boundary conditions is required. In the present study, we assumed that the vascular bed maintains a constant flow in both healthy and stenosed artery.

The flows were considered unsteady, laminar, and fully developed throughout the study section; hence, the velocity distribution in the inlet was set to be parabolic as expressed by (3):

$$
u=u_{\max }\left[1-\left(\frac{R}{R_{a}}\right)^{2}\right],
$$

where $R$ is the radial position and $R_{a}$ the inner radius of the artery.

Time-dependant velocity was taken from the literature for the left coronary artery during the cardiac cycle [33]. The waveform has a period $T=0.7 \mathrm{~s}$, where $0<t(\mathrm{sec})<0.2$ is systolic and $0.2<t(\mathrm{sec})<0.7$ is diastolic phase. The maximum velocity magnitude at resting condition varies between $0.15 \mathrm{~m} / \mathrm{s}$ and $0.25 \mathrm{~m} / \mathrm{s}$ for a coronary artery of $4 \mathrm{~mm}$ in diameter. The arterial flow at peak diastole in MBP is $102.2 \mathrm{~mL} / \mathrm{min}[34,35]$. For the outlet of the vessels, a traction-free boundary condition was imposed [36]. The Newtonian blood properties in this model are blood viscosity $\mu=0.0035$ Pa.s, and blood density $\rho=1060 \mathrm{Kg} / \mathrm{m}^{3}$, respectively [37].

\section{Results and Discussions}

Pulsatile hemodynamic analyses were carried out in healthy and stenotic bifurcations to study the influences of the constriction location on flow conditions and WSS distributions.

3.1. Flow Simulation Corresponding to Healthy Coronary Artery Bifurcation. A model of the healthy coronary artery bifurcation was developed and the corresponding velocity field and WSS distributions were investigated. The computed velocity field at peak diastole is presented in Figure 2.

Velocity profiles in the normal coronary artery bifurcation are skewed toward the carina, resulting in higher velocity along the inner walls and lower velocity along the outer walls.

For a Newtonian flow, WSS is the magnitude of the tangential shear forces acting on the wall by the fluid and is determined by the wall shear rate (gradient of velocity at the wall) multiplied by the viscosity of the fluid $(\tau=\mu \dot{\gamma})$. It is observed that, the gradient of velocity creates significant changes in the WSS on the inner and outer walls of MBD and SB. Distribution of WSS in the healthy artery at peak diastole is shown in Figure 3.

The distribution of WSS in Figure 3(a) shows the regions of high shear stress at the carina which is drastically greater than the WSS throughout the majority of the vessel walls.

The endothelial cells (lining the arterial walls) are exposed to shear stress variations due to periodic nature of blood flow. The physiological magnitude of the WSS ranges 


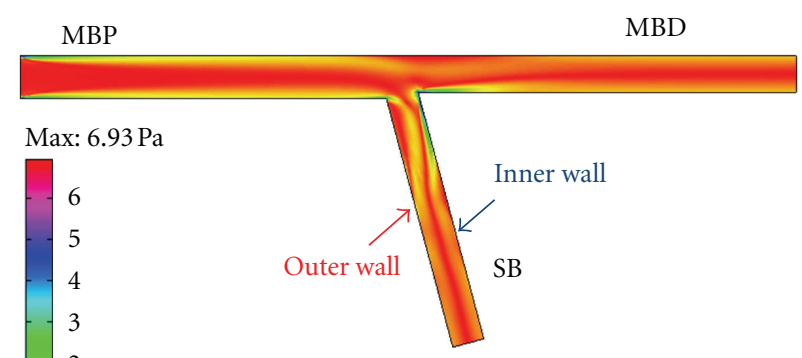

(a)

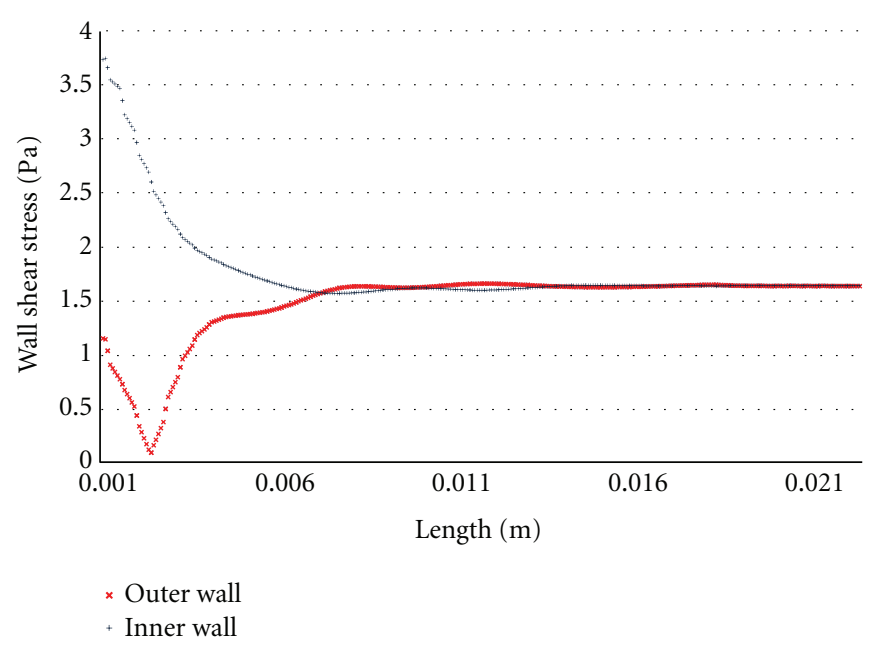

(b)

FIGURE 3: Distributions of WSS in the healthy coronary artery bifurcation (a) and WSS along the inner and outer walls of SB (b).

from 1 to $7 \mathrm{~Pa}$ in normal arteries [38]. Wall shear stress outside this range can potentially generate mechanisms that lead to vascular pathology. Lower values of WSS may contribute to the atherosclerotic process and therefore are considered "athero-prone," while WSS higher than this range may activate platelets and consequently "high-shear" induced thrombosis [38]. Prior in vitro and in vivo researches locate the atherosclerosis mostly within regions of disturbed blood flow, where WSS is below $<0.5 \mathrm{~Pa}$ [39]. The WSS values along the inner and outer walls depicted in Figure 3 indicate that a small portion of the luminal surface is exposed to such low WSS at peak diastole in the non-diseased bifurcation model. The larger value of the WSS at peak diastole occurs on the SB inner wall downstream the carina. The results indicate that on the SB, low WSS values occur on the outer wall. The low WSS distribution on the outer walls is in accordance with the localization of atherosclerotic lesions in these areas. Indeed, there is strong evidence that low WSS values are possibly correlated to the lesion localization [39-41].

3.2. Flow Simulation of Bifurcation Lesions $(1,1,1),(0,1$, $1)$, and $(1,0,1)$. Having discussed the flow condition and WSS distribution in healthy bifurcation, we next proceed to inspect the flow condition and WSS distribution in three lesion types of the Medina lesion classification (Figure 1). In clinical medicine, the severity of stenoses is commonly defined as the percentage of occlusion using diameter measurements: \% stenosis $=\left(d_{1}-d_{2}\right) / d_{1} * 100 \%$, where $d_{1}$ is the artery diameter and $d_{2}$ is the constricted diameter. As the disease advances, the percentage of stenosis also increases. The geometry of stenoses used in this simulation is the same geometry as Ahmed and Giddens used in their experimental work [42]. Symmetric moderate (50\%) and severe (75\%) stenoses with Gaussian symmetric surface morphology are imposed at the MBP, MBD, and SB, respectively.

The computational model and mechanical properties are built based on the coronary artery bifurcation detailed in

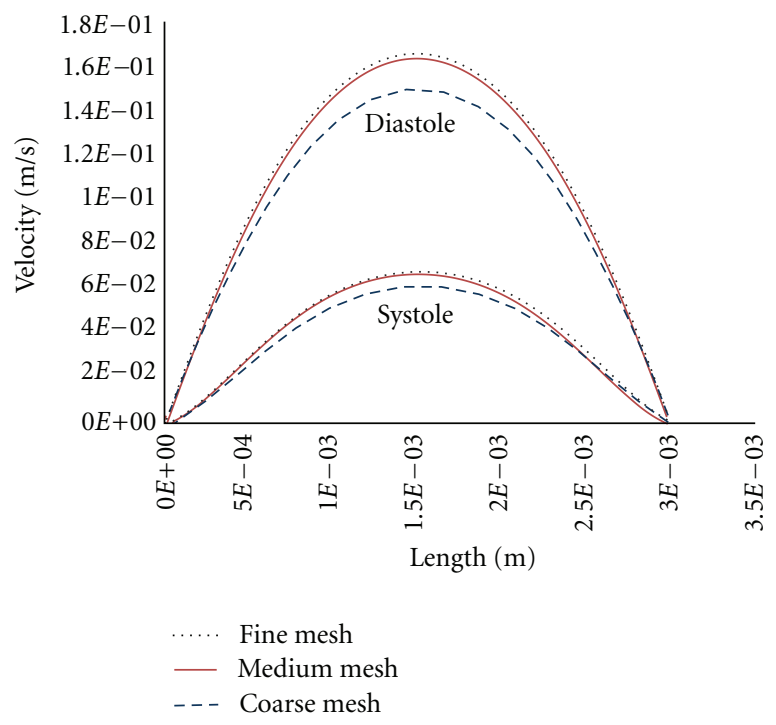

FIGURE 4: Velocity fields in peak systole and peak diastole in SB for three mesh densities.

section one. The obstructions in $\mathrm{MBP}, \mathrm{MBD}$, and $\mathrm{SB}$ are far from the outlet so that the flow can return to a nearly fully developed state and the outlet boundary condition does not influence activities occurring upstream.

For the flow modelling, mesh sensitivity analysis was carried out for bifurcation lesions $(1,1,1),(0,1,1)$, and $(1,0,1)$ for three different mesh densities and velocity fields, and WSS values were compared for different number of elements. Unsteady simulations were performed using a coarse, medium, and fine mesh of the geometry to estimate the mesh sensitivity for the arterial bifurcation geometry throughout the cardiac cycle. In bifurcation lesion $(1,1,1)$ with $50 \%$ stenosis, we used a coarse mesh (8665 elements); a medium mesh (13,097 elements); a fine mesh (19,625 elements). 

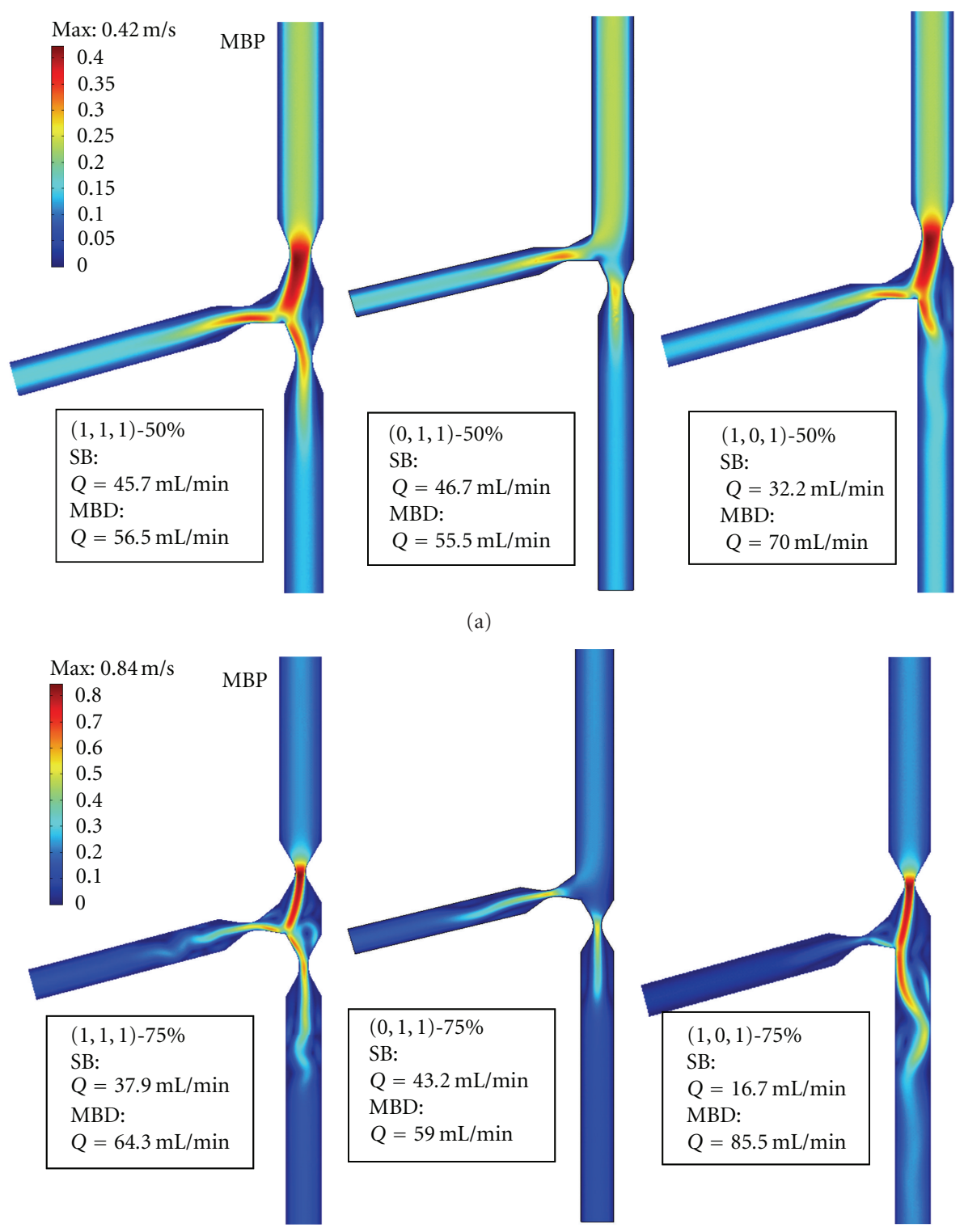

(b)

FIGURE 5: Maps of the velocity field magnitude and corresponding flow rates in 50\% (a) and 75\% (b) stenosed bifurcations.

Results were analyzed in term of the velocity profile and WSS along the MBD and SB. The velocity profiles and WSS for the coarse mesh density showed maximum $8 \%$ difference with the medium mesh. The results showed that the velocity field and WSS values have maximum $1 \%$ difference in the medium and fine mesh densities. Considering the negligible difference between medium and fine mesh densities and also the computing time, the medium mesh with mesh density of 13,097 elements was employed. For illustration, for bifurcation type $(1,1,1)$, we present the velocity fields in peak systole and peak diastole in SB for three mesh densities in Figure 4.

Transient simulations were performed for stenosed coronary artery bifurcations and the corresponding velocity fields and shear stress distributions were investigated. In Figure 5, the three representative snapshots of the magnitude of the velocity field for $50 \%$ and $75 \%$ stenoses are shown at peak diastole $(t=0.27 \mathrm{~s})$ for bifurcation lesion types $(1,1,1)$, $(0,1,1)$ and $(1,0,1)$ of Medina lesion classification.

The simulation results presented in Figure 5 shows a dramatically different velocity profile of the blood flow in the downstream of stenotic bifurcations and demonstrated the influence of the bifurcation lesion types on the blood flow pattern, such as flow separation and recirculation zones. The maximum velocity in bifurcation lesions with $50 \%$ and $75 \%$ stenoses are about $0.42 \mathrm{~m} / \mathrm{s}$ and $0.84 \mathrm{~m} / \mathrm{s}$, respectively which are related to the stenosed bifurcations with constriction in their MBP. Downstream the lesions, the flow decelerates and reverses near the walls due to the viscous effects. The bifurcation lesion types with greater stenoses (75\%), 


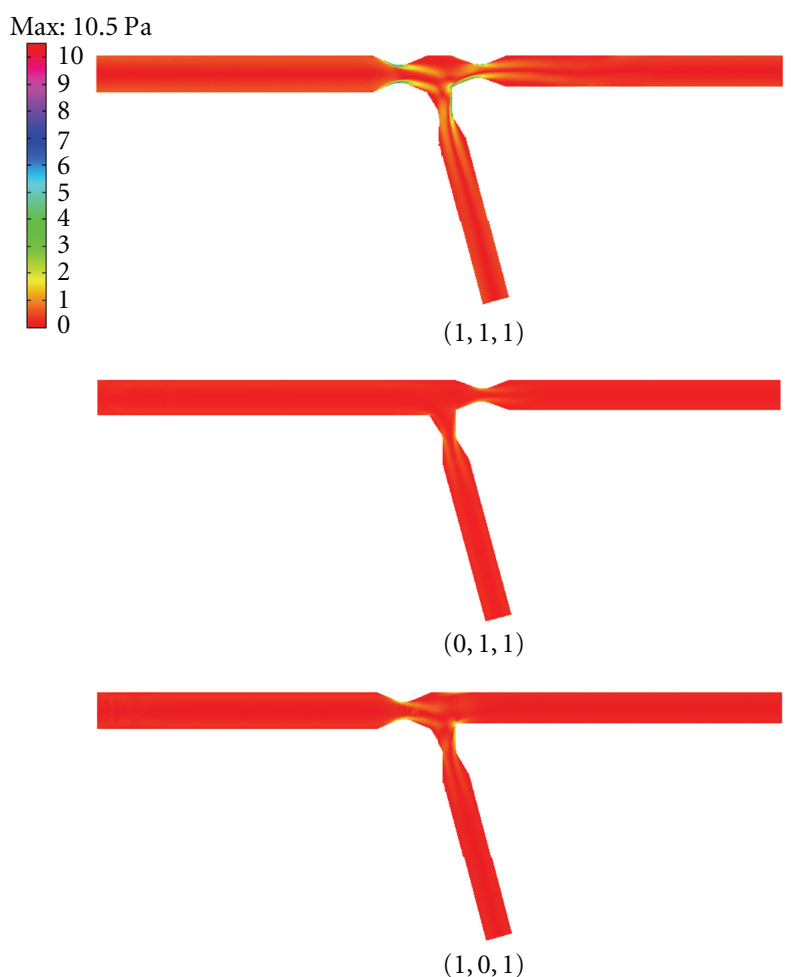

(a)

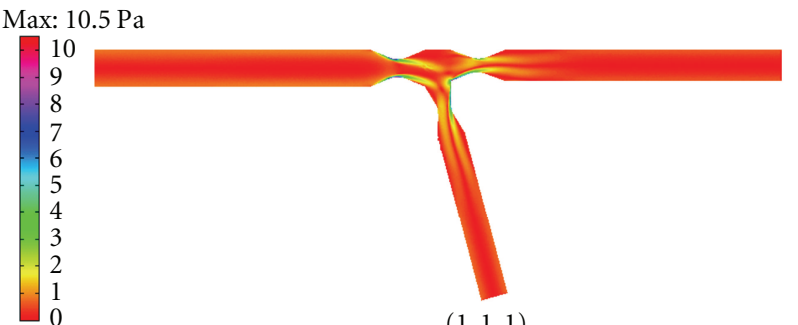

$(1,1,1)$

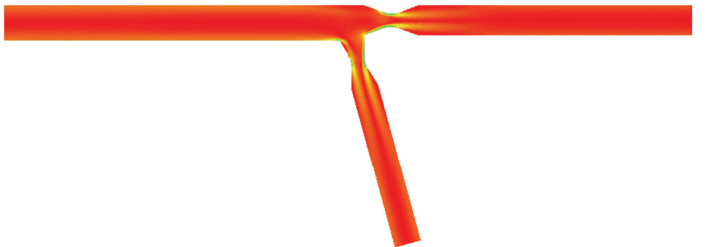

$(0,1,1)$

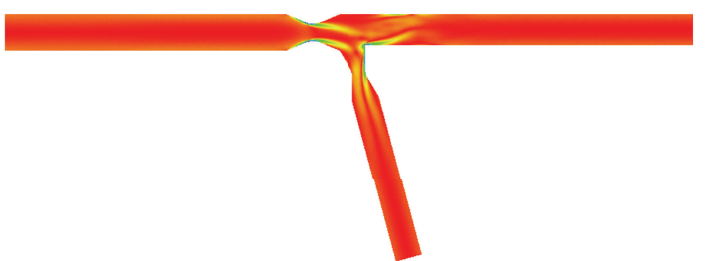

$(1,0,1)$

(b)

Figure 6: Wall shear stress at peak systole (a) and peak diastole (b) in true bifurcation lesions.

TABLE 1: The ratio of SB flow to MBP flow.

\begin{tabular}{lccc}
\hline $\begin{array}{l}\text { Diameter } \\
\text { reduction }\end{array}$ & $(1,1,1)$ & $(0,1,1)$ & $(1,0,1)$ \\
\hline \multirow{2}{*}{$50 \%$} & $\mathrm{Q}_{\mathrm{SB}} / \mathrm{Q}_{\mathrm{MBP}}=$ & $\mathrm{Q}_{\mathrm{SB}} / \mathrm{Q}_{\mathrm{MBP}}=$ & $\mathrm{Q}_{\mathrm{SB}} / \mathrm{Q}_{\mathrm{MBP}}=$ \\
& 0.44 & 0.45 & 0.31 \\
\hline \multirow{2}{*}{$75 \%$} & $\mathrm{Q}_{\mathrm{SB}} / \mathrm{Q}_{\mathrm{MBP}}=$ & $\mathrm{Q}_{\mathrm{SB}} / \mathrm{Q}_{\mathrm{MBP}}=$ & $\mathrm{Q}_{\mathrm{SB}} / \mathrm{Q}_{\mathrm{MBP}}=$ \\
& 0.37 & 0.42 & 0.16 \\
\hline
\end{tabular}

experience more flow separation, and bigger recirculation zones downstream the stenoses compare to bifurcation lesions with $50 \%$ stenoses. The results presented in Figure 5 also show that bifurcations with constriction in both MBD and $\mathrm{SB}(1,0,1)$ has the lowest magnitude of blood streaming into the SB. We calculated the ratio of the magnitude of the SB flow to the total MBP flow at peak diastole for all bifurcation lesions. The flow values were determined from converged simulation results and velocity profiles at $2.4 \mathrm{~cm}$ $\left(8 \mathrm{D}_{\mathrm{MBD}}\right)$ and $1.62 \mathrm{~cm}\left(6 \mathrm{D}_{\mathrm{SB}}\right)$ distal to the lesions in $\mathrm{MBD}$ and SB. As a consequence of presence or absence of lesions in MBD and MBP, various hemodynamic conditions occur in $\mathrm{SB}$ with the same percentage of stenosis (Figure 5). The ratio of maximal blood flow achievable in a stenotic SB to the maximal blood flow in MBP in various bifurcation lesion types for intermediate and severe stenoses are summarize in Table 1.

As it is described in Table 1, in bifurcation lesion $(1,0,1)$, the maximal diastolic SB flow in intermediate and severe stenoses is $31 \%$ and $16 \%$ of the total MBP flow. The results support that, the lower blood flow in the SB creates a crucial condition for this branch in bifurcation lesion $(1,0,1)$ compared to bifurcation lesions $(1,1,1)$, and $(0,1,1)$ respectively.

3.3. Wall Shear Stress (WSS) of the Bifurcation Lesion Types. WSS is one of the most important factors in developing atherosclerosis, and arterial branches are found more prone in formation of arterial plaques [20-23]. Although atherosclerosis is a disease affecting the vascular system as a whole, it has uneven distribution in SB and MBD with considerable differences for different bifurcation lesion types. Therefore, the WSS distribution is studied along the inner and outer walls of SB and MBD for true bifurcations $(1,1,1)$, $(0,1,1)$, and $(1,0,1)$ associated with the Medina lesion classification.

At peak diastolic point of a cardiac cycle, the WSS fields for true bifurcation lesions with 50\% stenoses are presented in Figure 6.

The distribution pattern shows that, at the MBP and upstream the flow divider, WSS is uniform. The peak values of the WSS are at the center of stenosis and at the carina of bifurcation. The WSS value at the inner and outer walls peaks at the center of stenosis and reaches the minimum in the post-stenosis region and then recovers gradually in the downstream stenosis along the arterial wall until it levels off. As mentioned, low WSS regions which are linked with 


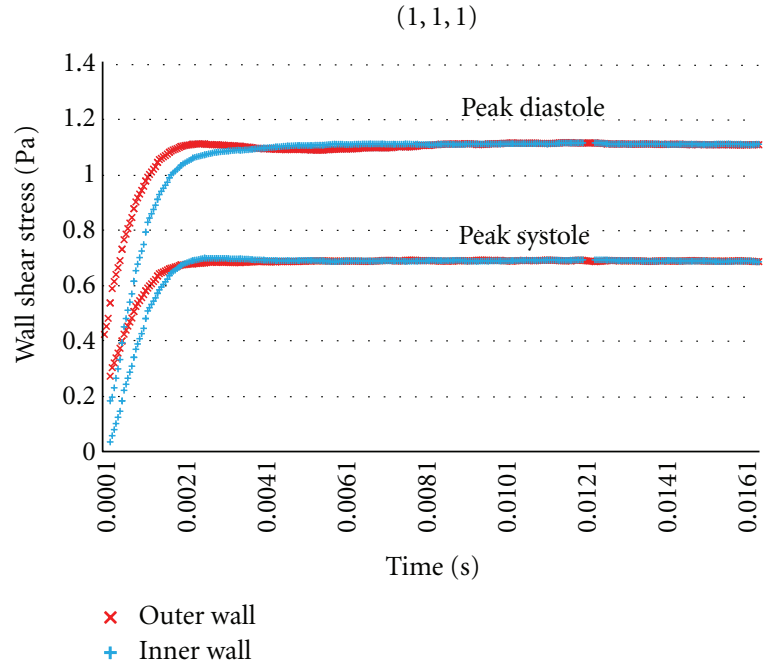

(a)

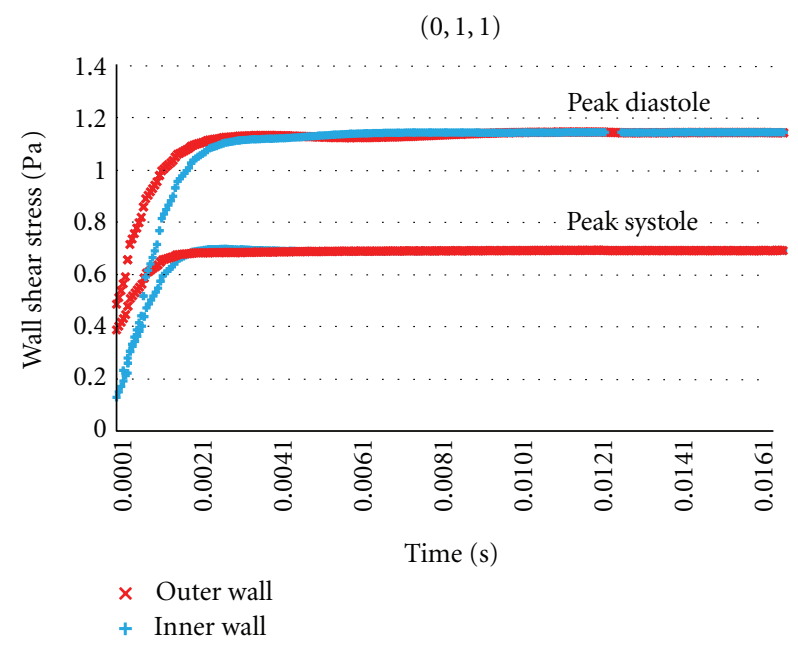

(b)

$(1,0,1)$

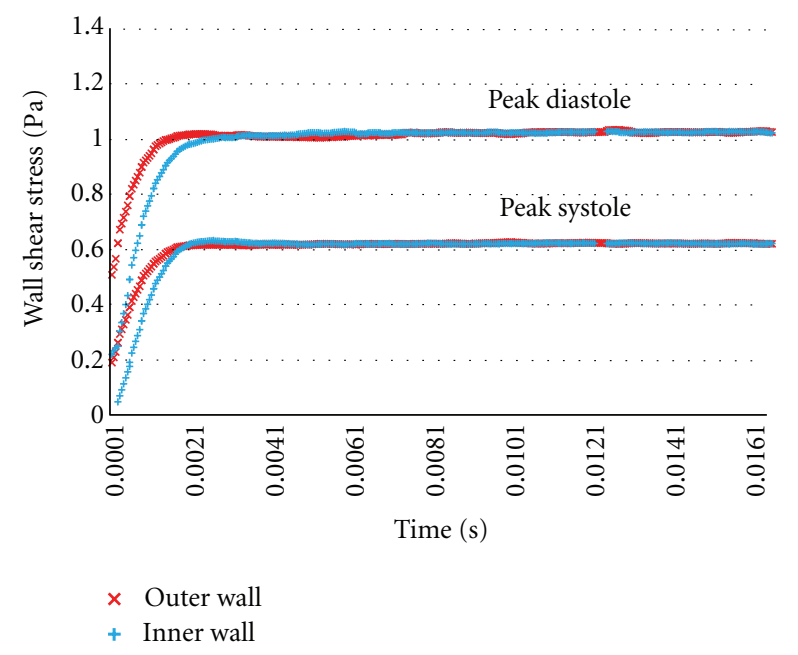

(c)

FIGURE 7: Wall shear stress on the outer and inner walls of SB and peak diastole.

atherosclerosis progression occur within the recirculation zone downstream the stenoses on the inner and outer walls.

Wall shear stress distribution on the inner and outer walls of SB downstream the stenosis is studied for bifurcation types $(1,1,1),(0,1,1)$, and $(1,0,1)$. The variation of WSS downstream the lesions on the outer and inner walls of SB at peak diastole are presented in Figure 7.

Comparing WSS values along the inner and outer walls between normal (Figure 3) and atherosclerotic bifurcations (Figure 7) revealed that the arteriosclerotic walls contain more regions exposed to low WSS values. In arteriosclerotic bifurcations, the magnitude of WSS on inner and outer walls of bifurcation type $(1,0,1)$ both in post stenotic region and along the arterial wall is smaller than the corresponding magnitudes in bifurcation types $(1,1,1)$ and $(0,1,1)$.

Low WSS regions which are associated with atherosclerosis progression occur within the recirculation zone downstream the stenoses. In bifurcation lesions with stenosis in their SB, low WSS regions were observed in the poststenosis regions is the $\mathrm{SB}$. We now examine the time-dependant behavior of the WSS in the poststenosis region on the outer wall of SB. For this purpose, three consecutive points (A, B, and $\mathrm{C}$ ) are considered downstream the stenosis on the outer wall. Point $\mathrm{A}$ is located $0.1 \mathrm{~mm}$ downstream the stenosis. The $\mathrm{A}-\mathrm{B}$ and $\mathrm{B}-\mathrm{C}$ distances are $1.5 \mathrm{~mm}$. The temporal variation of WSS downstream the lesions, at three consecutive points on the outer wall of SB for true bifurcation are presented in Figure 8.

The change of WSS throughout the cardiac cycle is highly correlated to flow velocities. The WSS characterizes the forces that longitudinally act on the vessel wall. At peak systole and peak diastole, when the blood flow parallel to the wall is fast, these forces are higher. At each individual point on the SB wall, the maximum value of WSS in all bifurcation types is related to the peak systolic and peak diastolic of a cardiac cycle. The maximum value of WSS for bifurcation lesion 

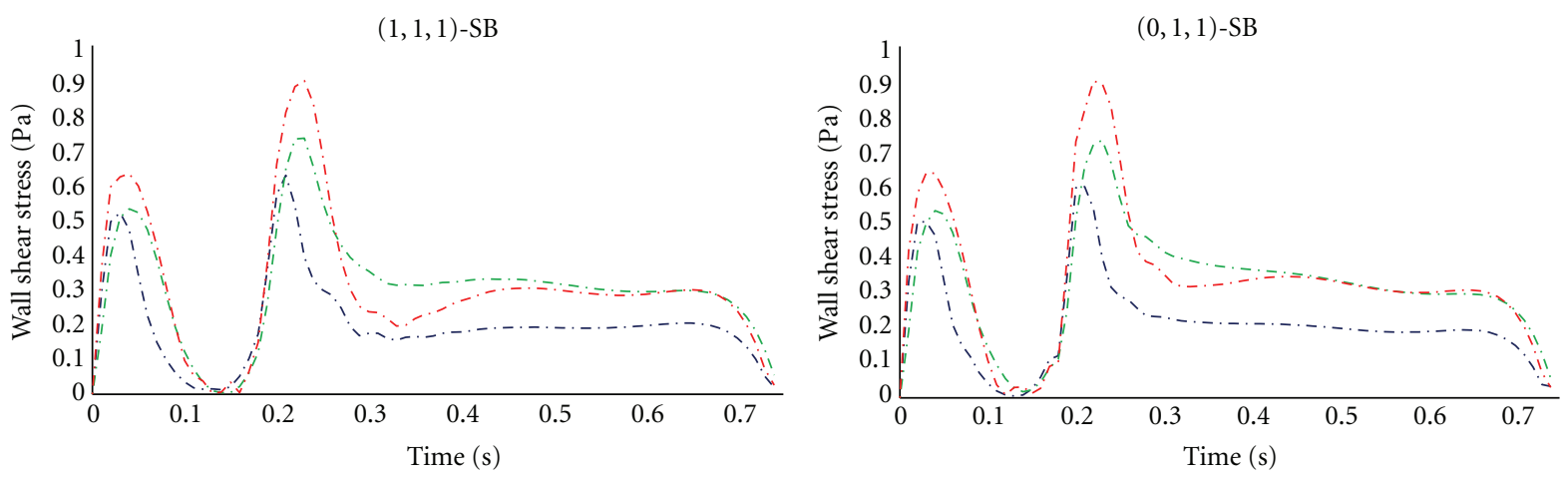

$\ldots$ (A)
$\cdots$ (B)
$\cdots$ (C)

$\cdot-\cdot-(\mathrm{A})$

$\cdots(\mathrm{B})$

$-.-(\mathrm{C})$

(a)

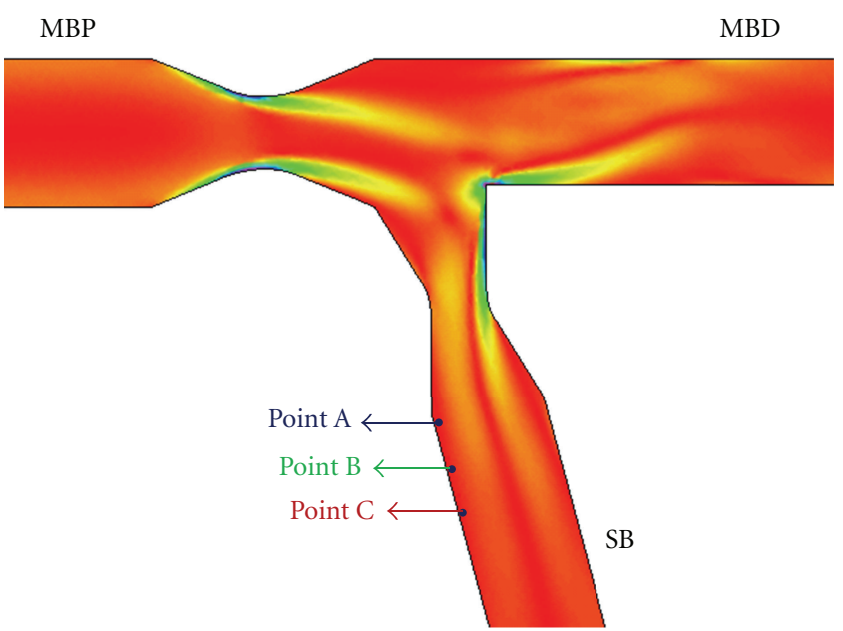

(c)

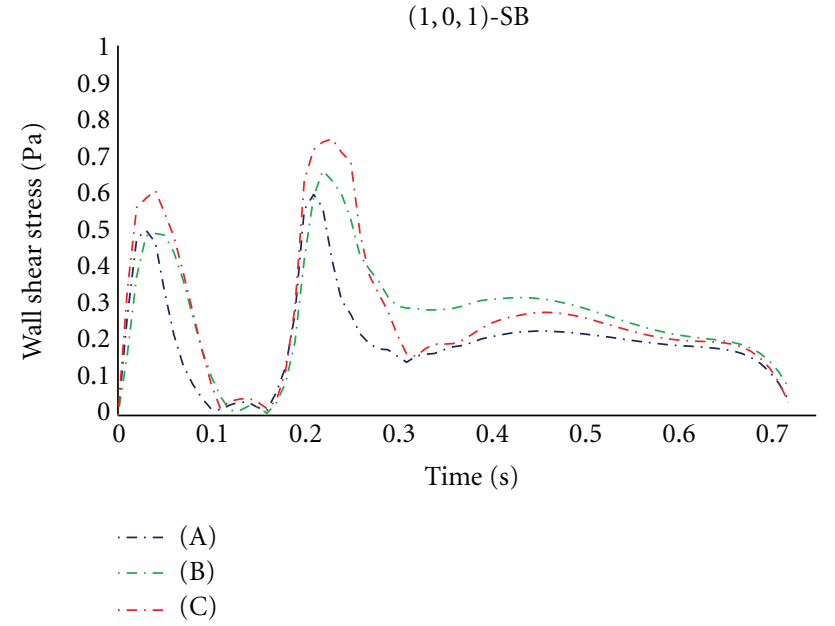

(d)

FIgURE 8: Temporal WSS at three points on the outer walls of SB for true bifurcation lesions.

types $(1,1,1)$ and $(0,1,1)$ at peak systolic and diastolic points are $0.65 \mathrm{~Pa}$ and $0.9 \mathrm{~Pa}$. The corresponding WSS for lesion type $(1,0,1)$ is $0.58 \mathrm{~Pa}$ and $0.75 \mathrm{~Pa}$ revealing that the minimum magnitude of WSS is related to the lesion type ( 1 , $0,1)$. The low values of WSS are mostly in the deceleration phase which possess two third of a cardiac cycle duration.

To assess how WSS pattern changes in MBD along the entire wall in all time steps of a cardiac cycle, the mean WSS values along the inner and outer walls downstream the lesions are computed for 68 nodes in bifurcation lesion types $(1,1,1)$ and $(0,1,1)$ and for 78 nodes in bifurcation lesion type $(1,0,1)$. The mean WSS values are determined at each time step and mean WSS values along the inner and outer walls of MBD during a cardiac cycle are presented in Figure 9.

The distribution pattern of WSS on the MBD reveals that, most parts of the luminal surface are exposed to WSS within the physiological range. Comparisons of the WSS distributions in bifurcation lesion types $(1,1,1),(0,1,1)$, and $(1,0,1)$ shows that during the diastolic period, arterial regions downstream bifurcation lesion types $(1,1,1),(0,1$, 1) exposes to lower magnitudes of WSS.

As another important hemodynamic parameter, WSS is studied in true bifurcation lesions. Predictions of WSS distribution downstream the stenosis is useful in the understanding of the effects of disturbed flow on endothelial cells and blood elements near the wall and can be used to support medical decision.

The results of the present study should be interpreted within the constraints of certain limitations. First, the geometries used in this work are a series of idealized two dimensional axisymmetric stenoses in idealized arterial bifurcation. In our problem, stenoses configurations and percentages are fixed while in reality they may vary and occur in different places along the vessel. Another geometric limitation of the present study is the fact that we do not take the composition of the compliance of the vessels; all the vessels are considered as rigid wall boundaries. The simulations do not take into consideration the non-Newtonian property of the blood. Indeed, while blood behaves generally like a Newtonian fluid 


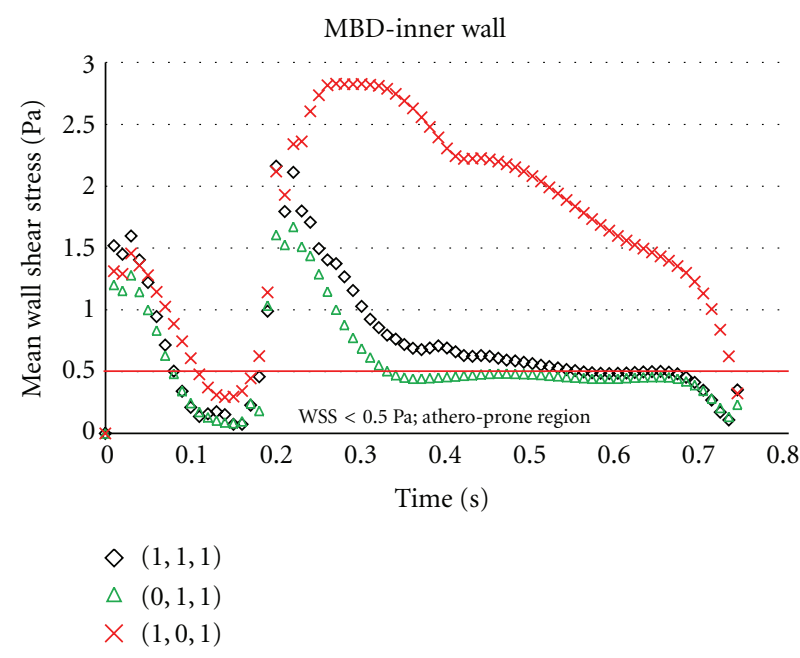

(a)

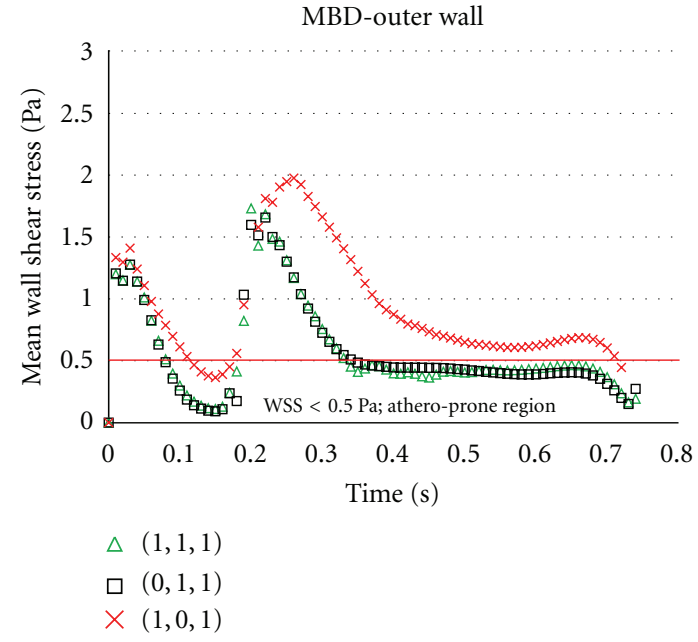

(b)

FIGURE 9: Mean WSS value along the inner and outer walls of MBD during a cardiac cycle.

at high shear rates, the non-Newtonian properties may affect the blood flow patterns and shear stress results at low shear rate regions. In addition, our geometric model is based on a representation of a straight blood vessel, while they may be curved in reality. The vessel curvature can have significant effect on the skewness of the velocity profile and the general behavior of the flow.

\section{Conclusion}

Most experimental and numerical studies of pulsatile flow through stenotic arteries have been performed assuming a simple vessel and there are no studies of hemodynamic changes associated with various bifurcation lesion types to date especially in the context of the Medina lesion classification. The present study is motivated by the need to understand the flow condition and WSS distributions in various bifurcation lesion types in a coronary artery bifurcation. We have investigated the flow ratio and time-dependence patterns of WSS resulting from the numerical simulation of pulsating hemodynamic flows in healthy and stenosed coronary artery bifurcations. Various stenoses configurations were considered according to Medina lesion classification and a detailed numerical result for time-dependent WSS distributions that may be involved in lesion initiation and progression is highlighted. The main results of this study are that different bifurcation type determines different flow ratio and WSS distributions in SB. In detail, the ratio of SB flow to MBP flow was less in both $50 \%$ and $75 \%$ for $(1,0,1)$ bifurcation lesion type compared to $(0,1,1)$ and $(1,1,1)$. Examinations of the WSS distribution in true bifurcation lesions showed that on the $\mathrm{SB}$, in terms of athero-prone regions, the lesion type $(1,1,1)$ is not likely the worst case because the results support that lesion type $(1,0,1)$ resulted in lower values of WSS on both inner and outer walls especially in the deceleration phase of the cardiac cycle. The results indicated that in the bifurcation lesion types, the flow condition and WSS distribution in SB are influenced by the lesion morphologies which cannot be fully assessed by quantitative coronary angiographic parameters.

\section{References}

[1] I. H. Tanboga, M. Ekinci, T. Isik, M. Kurt, A. Kaya, and S. Sevimli, "Reproducibility of syntax score: from core lab to real world," Journal of Interventional Cardiology, vol. 24, no. 4, pp. 302-306, 2011.

[2] F. Zhang, L. Dong, and J. Ge, "Simple versus complex stenting strategy for coronary artery bifurcation lesions in the drugeluting stent era: a meta-analysis of randomised trials," Heart, vol. 95, no. 20, pp. 1676-1681, 2009.

[3] M. Pan, J. Suárez De Lezo, A. Medina et al., "Simple and complex stent strategies for bifurcated coronary arterial stenosis involving the side branch origin," American Journal of Cardiology, vol. 83, no. 9, pp. 1320-1325, 1999.

[4] E. Jorgensen and S. Helqvist, "Stent treatment of coronary artery bifurcation lesions," European Heart Journal, vol. 28, no. 4, pp. 383-385, 2007.

[5] I. Iakovou, L. Ge, and A. Colombo, "Contemporary stent treatment of coronary bifurcations," Journal of the American College of Cardiology, vol. 46, no. 8, pp. 1446-1455, 2005.

[6] J. Popma, M. Leon, and E. J. Topol, Atlas of Interventional Cardiology, Saunders, Philadelphia, Pa, USA, 1994.

[7] A. M. Spokojny and T. M. Sanborn, "The bifurcation lesion," in Strategic Approaches in Coronary Intervention, S. G. Ellis and D. R. Holmes, Eds., p. 288, Williams and Wilkins, Baltimore, Md, USA, 1996.

[8] T. Lefèvre, Y. Louvard, M. C. Morice et al., "Stenting of bifurcation lesions: classification, treatments, and results," Catheterization and Cardiovascular Interventions, vol. 49, pp. 274-283, 2000.

[9] R. D. Safian, "Bifurcation lesions," in Manual of Interventional Cardiology, R. D. Safian and M. Freed, Eds., pp. 221-236, Physicians' Press, Royal Oak, Mich, USA, 2001.

[10] G. Sianos, M. A. Morel, and A. P. Kappetein, "The SYNTAX score: an angiographic tool grading the complexity of 
coronary artery disease," Eurointervention, vol. 1, no. 2, pp. 219-227, 2005.

[11] M. R. Movahed and C. T. Stinis, "A new proposed simplified classification of coronary artery bifurcation lesions and bifurcation interventional techniques," Journal of Invasive Cardiology, vol. 18, no. 5, pp. 199-204, 2006.

[12] A. Medina, J. Suarez de Lezo, and M. A. Pan, "A new classification of coronary bifurcation lesions," Revista Espanola de Cardiologia, vol. 59, no. 2, p. 183, 2006.

[13] T. Asakura and T. Karino, "Flow patterns and spatial distributions of atherosclerotic lesions in human coronary arteries," Circulation Research, vol. 66, no. 4, pp. 1045-1066, 1990.

[14] B. I. Tropea, S. Glagov, and C. K. Zarins, Hemodynamics and Atherosclerosis, Futura, Armonk, NY, USA, 1997.

[15] C. G. Caro, J. M. Fitz-Gerald, and R. C. Schroter, "Arterial wall shear and distribution of early atheroma in man," Nature, vol. 223, pp. 1159-1161, 1969.

[16] A. M. Malek, S. L. Alper, and S. Izumo, "Hemodynamic shear stress and its role in atherosclerosis," Journal of the American Medical Association, vol. 282, no. 21, pp. 2035-2042, 1999.

[17] P. H. Stone, A. U. Coskun, Y. Yeghiazarians et al., "Prediction of sites of coronary atherosclerosis progression: in vivo profiling of endothelial shear stress, lumen, and outer vessel wall characteristics to predict vascular behavior," Current Opinion in Cardiology, vol. 18, no. 6, pp. 458-470, 2003.

[18] M. A. J. Gimbrone, J. N. Topper, T. Nagel, K. R. Anderson, and G. Garcia-Cardena, "Endothelial dysfunction, hemodynamic forces, and atherogenesis," Annals of the New York Academy of Sciences, vol. 902, pp. 230-239, 2000.

[19] K. S. Cunningham and A. I. Gotlieb, "The role of shear stress in the pathogenesis of atherosclerosis," Laboratory Investigation, vol. 85, no. 1, pp. 9-23, 2005.

[20] K. C. Koskinas, C. L. Feldman, Y. S. Chatzizisis et al., "Natural history of experimental coronary atherosclerosis and vascular remodeling in relation to endothelial shear stress: a serial, in vivo intravascular ultrasound study," Circulation, vol. 121, no. 19, pp. 2092-2101, 2010.

[21] C. Cheng, D. Tempel, R. van Haperen et al., "Atherosclerotic lesion size and vulnerability are determined by patterns of fluid shear stress," Circulation, vol. 113, no. 23, pp. 2744-2753, 2006.

[22] D. N. Ku, D. P. Giddens, C. K. Zarins, and S. Glagov, "Pulsatile flow and atherosclerosis in the human carotid bifurcation. Positive correlation between plaque location and low and oscillating shear stress," Arteriosclerosis, vol. 5, no. 3, pp. 293302, 1985.

[23] S. Fabregues, K. Baijens, R. Rieu, and P. Bergeron, "Hemodynamics of endovascular prostheses," Journal of Biomechanics, vol. 31, no. 1, pp. 45-54, 1997.

[24] C. von Birgelen, S. G. Airiian, G. S. Mintz et al., "Variations of remodeling in response to left main atherosclerosis assessed with intravascular ultrasound in vivo," American Journal of Cardiology, vol. 80, no. 11, pp. 1408-1413, 1997.

[25] J. B. Hermiller, C. E. Buller, A. N. Tenaglia et al., "Unrecognized left main coronary artery disease in patients undergoing interventional procedures," American Journal of Cardiology, vol. 71, no. 2, pp. 173-176, 1993.

[26] T. C. Gerber, R. Erbel, G. Gorge, J. Ge, H. J. Rupprecht, and J. Meyer, "Extent of atherosclerosis and remodeling of the left main coronary artery determined by intravascular ultrasound," American Journal of Cardiology, vol. 73, no. 9, pp. 666-671, 1994.

[27] Z. Kaimkhani, M. Ali, and A. M. A. Faruqui, "Coronary artery diameter in a cohort of adult Pakistani population," Journal of the Pakistan Medical Association, vol. 54, no. 5, pp. 258-261, 2004.

[28] C. Godino, R. Al-Lamee, C. La Rosa et al., "Coronary left main and non-left main bifurcation angles: how are the angles modified by different bifurcation stenting techniques?" Journal of Interventional Cardiology, vol. 23, no. 4, pp. 382393, 2010.

[29] D. G. Rizik, K. J. Klassen, and J. B. Hermiller, "Bifurcation CAD: current techniques and future directions: morphology of the bifurcation," Journal of Invasive Cardiology, vol. 20, no. 2, pp. 82-90, 2008.

[30] S. H. Na, B. K. Koo, J. C. Kim et al., "Evaluation of local flow conditions in jailed side branch lesions using computational fluid dynamics," Korean Circulation Journal, vol. 41, no. 2, pp. 91-96, 2011.

[31] Y. Louvard, A. Medina, and G. Stankovic, "Classification of coronary artery bifurcation lesions and treatments," Eurointervention, vol. 6, supplement J, pp. 31-35, 2010.

[32] J. F. Ladisa, J. I. Guler, L. E. Olson et al., "Three-dimensional computational fluid dynamics modeling of alterations in coronary wall shear stress produced by stent implantation," Annals of Biomedical Engineering, vol. 31, no. 8, pp. 972-980, 2003.

[33] I. Faik, R. Mongrain, R. L. Leask, J. Rodes-Cabau, E. Larose, and O. F. Bertrand, "Time-dependent 3D simulations of the hemodynamics in a stented coronary artery," Biomedical Materials, vol. 2, pp. S28-S37, 2007.

[34] Z. D. Zhang, M. Svendsen, J. S. Choy et al., "New method to measure coronary velocity and coronary flow reserve," American Journal of Physiology, vol. 301, no. 1, pp. H21-H28, 2011.

[35] W. G. Hundley, R. A. Lange, G. D. Clarke et al., "Assessment of coronary arterial flow and flow reserve in humans with magnetic resonance imaging," Circulation, vol. 93, no. 8, pp. 1502-1508, 1996.

[36] J. S. Stroud, S. A. Berger, and D. Saloner, "Numerical analysis of flow through a severely stenotic carotid artery bifurcation," Journal of Biomechanical Engineering, vol. 124, no. 1, pp. 9-20, 2002.

[37] N. Benard, R. Perrault, and D. Coisne, "Computational approach to estimating the effects of blood properties on changes in intra-stent flow," Annals of Biomedical Engineering, vol. 34, no. 8, pp. 1259-1271, 2006.

[38] Y. S. Chatzizisis, M. Jonas, A. U. Coskun et al., "Prediction of the localization of high-risk coronary atherosclerotic plaques on the basis of low endothelial shear stress-an intravascular ultrasound and histopathology natural history study," Circulation, vol. 117, no. 8, pp. 993-1002, 2008.

[39] B. Fox, K. James, B. Morgan, and A. Seed, "Distribution of fatty and fibrous plaques in young human coronary arteries," Atherosclerosis, vol. 41, no. 2-3, pp. 337-347, 1982.

[40] C. Velican and D. Velican, "Incidence, topography and lightmicroscopic feature of coronary atherosclerotic plaques in adults 26-35 years old," Atherosclerosis, vol. 35, no. 1, pp. 111$122,1980$.

[41] G. Nakazawa, S. K. Yazdani, A. V. Finn, M. Vorpahl, F. D. Kolodgie, and R. Virmani, "Pathological findings at bifurcation lesions: the impact of flow distribution on atherosclerosis and arterial healing after stent implantation," Journal of the American College of Cardiology, vol. 55, no. 16, pp. 1679-1687, 2010.

[42] S. A. Ahmed and D. P. Giddens, "Velocity measurements in steady flow through axisymmetric stenoses at moderate Reynolds numbers," Journal of Biomechanics, vol. 16, no. 7, pp. 505516,1983 

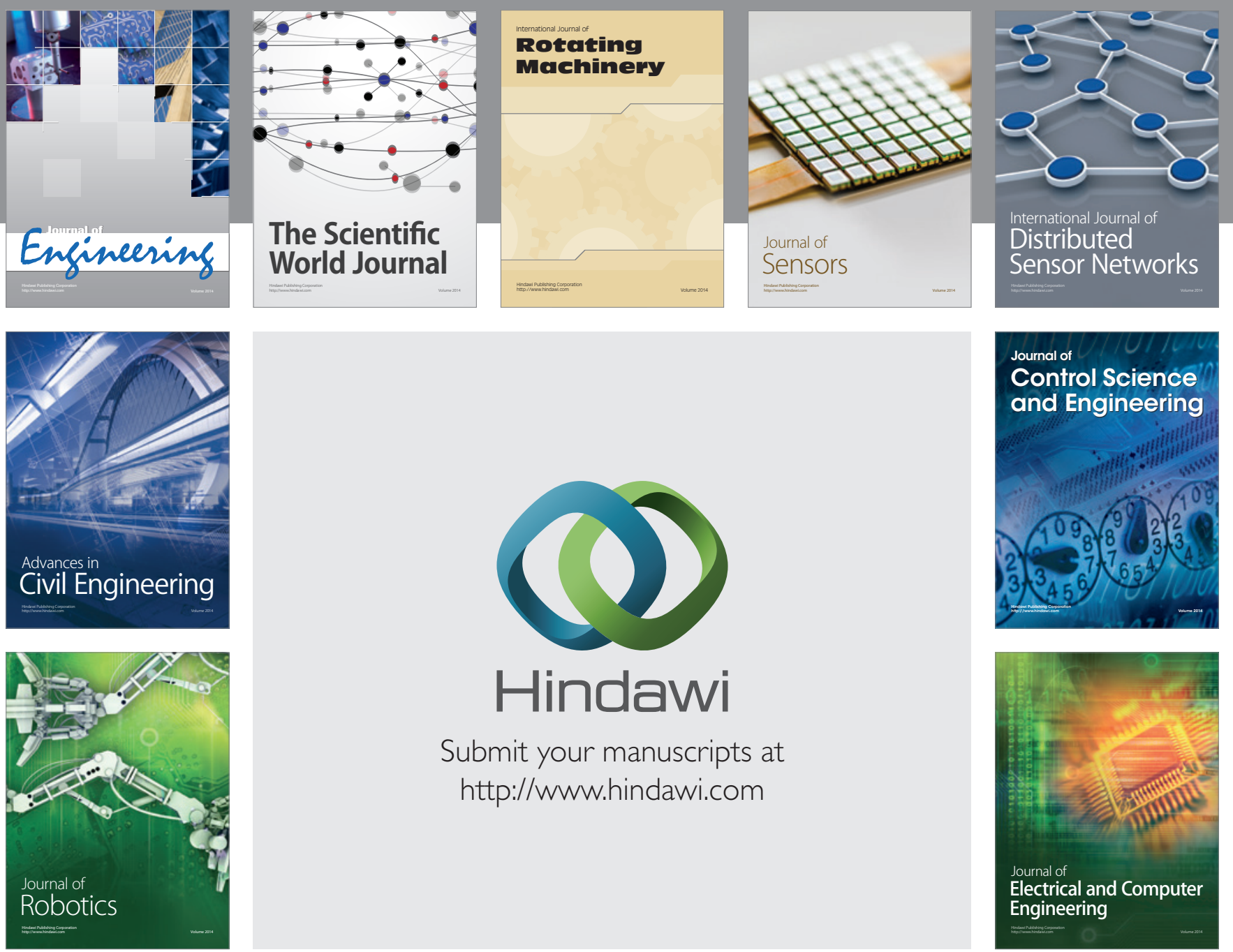

Submit your manuscripts at

http://www.hindawi.com
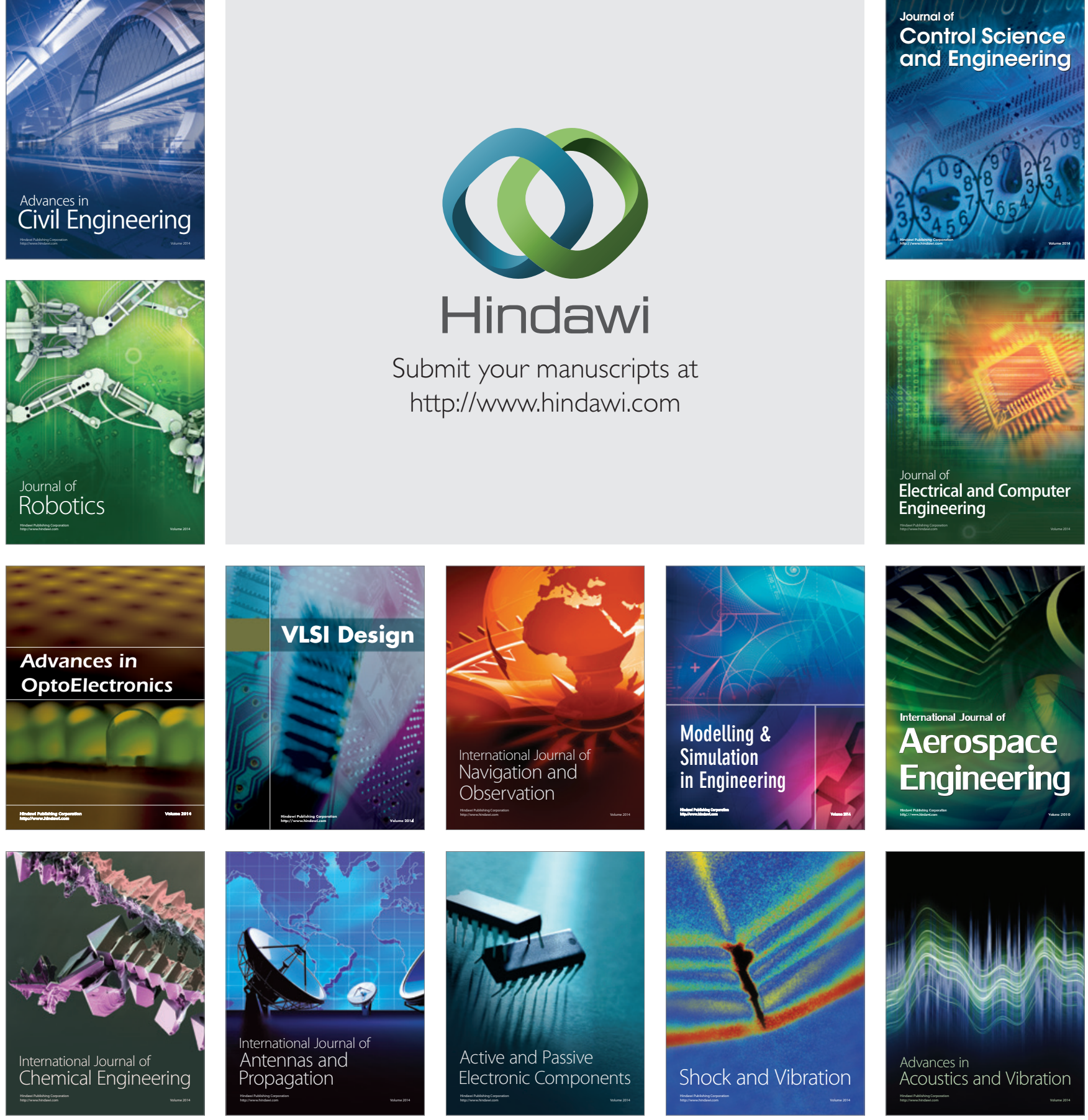